\title{
Investigation of the Interaction of Polar Molecules on Graphite Surface: Prediction of Isosteric Heat of Adsorption at Zero Surface Coverage
}

\author{
Wu Fan, Anutosh Chakraborty ${ }^{*}$ \\ School of Mechanical and Aerospace Engineering, Nanyang Technological University \\ 50 Nanyang Avenue, Singapore 639798, Republic of Singapore \\ *Corresponding author: E-mail: AChakraborty@,ntu.edu.sg, Tel: +65-6790-4222
}

\section{Multipole Expansion of Energy}

The energy of uncharged molecule in static electric filed can be expressed by multipole expansion formulation ${ }^{1,2}$

$$
\begin{aligned}
\mathrm{U}=\mathrm{U}^{0}-\boldsymbol{\mu}_{\alpha} \boldsymbol{F}_{\alpha} & -\frac{1}{3} \boldsymbol{\Theta}_{\alpha \beta} \boldsymbol{F}_{\alpha \beta}-\frac{1}{15} \boldsymbol{\Omega}_{\alpha \beta \gamma} \boldsymbol{F}_{\alpha \beta \gamma}-\frac{1}{105} \boldsymbol{\Phi}_{\alpha \beta \gamma \delta} \boldsymbol{F}_{\alpha \beta \gamma \delta}+\cdots \\
& -\frac{1}{2} \boldsymbol{\alpha}_{\alpha \beta} \boldsymbol{F}_{\alpha} \boldsymbol{F}_{\beta}-\frac{1}{3} \boldsymbol{A}_{\alpha, \beta \gamma} \boldsymbol{F}_{\alpha} \boldsymbol{F}_{\beta \gamma \delta}-\frac{1}{6} \boldsymbol{C}_{\alpha \beta, \gamma \delta} \boldsymbol{F}_{\alpha \beta} \boldsymbol{F}_{\gamma \delta}-\frac{1}{15} \boldsymbol{E}_{\alpha, \beta \gamma \delta} \boldsymbol{F}_{\alpha} \boldsymbol{F}_{\beta \gamma \delta \delta}+\cdots \\
& -\frac{1}{6} \boldsymbol{\beta}_{\alpha \beta \gamma} \boldsymbol{F}_{\alpha} \boldsymbol{F}_{\beta} \boldsymbol{F}_{\gamma}-\frac{1}{6} \boldsymbol{B}_{\alpha \beta, \gamma \delta} \boldsymbol{F}_{\alpha} \boldsymbol{F}_{\beta} \boldsymbol{F}_{\gamma \delta}+\cdots \\
& -\frac{1}{24} \boldsymbol{\gamma}_{\alpha \beta \gamma \delta} \boldsymbol{F}_{\alpha} \boldsymbol{F}_{\beta} \boldsymbol{F}_{\gamma} \boldsymbol{F}_{\delta}+\cdots
\end{aligned}
$$

where $\mathrm{U}^{0}$ is the energy of free molecule, $\boldsymbol{\mu}_{\alpha}, \boldsymbol{\Theta}_{\alpha \beta}, \boldsymbol{\Omega}_{\alpha \beta \gamma}$ and $\boldsymbol{\Phi}_{\alpha \beta \gamma \delta}$ are dipole, quadrupole, octupole and hexadecapole moment, $\boldsymbol{\alpha}_{\alpha \beta}, \boldsymbol{\beta}_{\alpha \beta \gamma}$ and $\boldsymbol{\gamma}_{\alpha \beta \gamma \delta}$ are dipole polarizabilities, $\boldsymbol{A}_{\alpha, \beta \gamma}$, $\boldsymbol{C}_{\alpha \beta, \gamma \delta}, \boldsymbol{E}_{\alpha, \beta \gamma \delta}$ and $\boldsymbol{B}_{\alpha \beta, \gamma \delta}$ are the quadrupole polarizabilities, $\boldsymbol{F}$ with subscripts are electric field, electric field gradient (EFG), etc. depends on the number of groups of subscripts. By applying the specific reduction strategies on equation (S1) based on the multipole properties of the molecule ${ }^{3}$, the multipole expression is suitable for the calculation of interaction energy of molecules. 


\section{Reduced Multipole Expansion of Energy}

Taking Lennard-Jones (LJ) and quadrupole-quadrupole interaction into consideration, Pople ${ }^{4}$ modified the potential and is given by,

$$
\mathrm{U}=\mathrm{u}_{\mathrm{LJ}}+u_{\text {quadrupole-quadrupole }}
$$

Considering Keesom Force between dipolar molecules, Keesom ${ }^{5}$ also proposed the improved intermolecular potential model as

$$
\mathrm{U}=\mathrm{U}_{\mathrm{HS}}+\mathrm{U}_{\text {dipole-dipole }} \text { or } \mathrm{U}=\mathrm{U}_{\mathrm{HS}}+\mathrm{U}_{\text {quadrupole-quadrupole }}
$$

For non-polar molecules, the interaction potential can be expressed by LJ potential sufficiently while electrostatic and induction effect cannot be neglected for polar molecules. Therefore, by combining the Lenard-Jones, electrostatic and induction potential $\mathrm{U}_{\mathrm{LJ}}, \mathrm{U}_{\mathrm{E}}, \mathrm{U}_{\mathrm{I}}$, the total interaction potential $\mathrm{U}_{\mathrm{mM}}$ is proposed to obtain more complete description of interaction potential for polar molecules.

$$
\mathrm{U}_{\mathrm{mM}}=\mathrm{U}_{\mathrm{LJ}}+\mathrm{U}_{\mathrm{E}}+\mathrm{U}_{\mathrm{I}}
$$

\subsection{Lenard Jones interaction potential}

L-J potential model is described in the main text of the paper.

\subsection{Electric Field and Energy of multipole}

\section{Potential of dipole in static electric field}

The potential of permanent dipole in electric field $\mathbf{F}$ is,

$$
\mathrm{U}=-\boldsymbol{\mu} \cdot \mathbf{F}
$$

A permanent dipole placed in static electric field will induce a temporary dipole with the same dipole moment and opposite direction. In the non-liner regime, the induced dipole moment $\boldsymbol{\mu}^{\mathrm{I}}$ is,

$$
\boldsymbol{\mu}^{\mathrm{I}}=\boldsymbol{\alpha} \cdot \mathbf{F},
$$


where $\boldsymbol{\alpha}$ is the dipole moment polarizability tensor of an acceptor of electric field and $\mathbf{F}$ is the exerted static electric field. Due to the induced dipole instead of a permanent one, the induction potential $U_{I}$ is,

$$
\mathrm{U}_{\mathrm{I}}=-\frac{1}{2} \boldsymbol{\mu}^{\mathrm{I}} \cdot \mathbf{F}
$$

The Debye Force between dipole and induced dipole adds to the intermolecular potential when non-zero dipole moment exists in the interaction pair. Taking this Induction Effect into account, the interaction potential $U_{I}$ is,

$$
\mathrm{U}=\mathrm{U}_{\mathrm{I}}=-\frac{1}{2} \boldsymbol{\alpha} \cdot \mathbf{F}^{2}
$$

\section{Electric field generated by a dipole}

Generally, the applied approximate equation for electric potential is valid for large distance. The dipole moment of water molecule is $\mathrm{p}=1.85 \mathrm{D}$ and the equivalent displacement is $\mathrm{d}=\mathrm{p} / \mathrm{q}=$ $1.85 \times\left(3.33564 \times 10^{-30} \mathrm{C} \cdot \mathrm{m}\right) /\left(0.82 \times 1.6 \times 10^{-19} \mathrm{C}\right)=4.7 \times 10^{-11} \mathrm{~m}=0.47 \AA \quad$ given that $\mathrm{qO}=-0.82 \mathrm{e}$. In the calculation of interaction potential for water, the cut-off distance of $\mathrm{z}=2.5 \AA$, which is more than 5 times of the displacement of the dipole. Therefore, the expression used for electric potential of dipole moment is considered as an acceptable approximation even for the minimum separation. Meanwhile, the accuracy of the expression will increase as the adsorbate-adsorbent separation $\mathrm{z}$ increases because the error of this approximation is inverse proportional to $\mathrm{z}^{2}$, which means the rest $\mathrm{U}(\mathrm{z})$ values on the potential curves are more reliable than the acceptable $U\left(\mathrm{z}_{\text {cut-off }}\right)^{6}$. Lastly, the final result reveals the fact that even for polar molecules the LJ potential is still the dominant part in the interaction potential and this also 
moderates the approximation of electric potential expressions. The electric potential of elementary charge in the electric field excited by a permanent dipole $\boldsymbol{\mu}$ at the distance $\mathbf{r}$ is,

$$
\phi_{\text {dipole }}(\mathbf{r})=\frac{1}{4 \pi \varepsilon_{0}} \frac{\mathbf{r} \cdot \boldsymbol{\mu}}{\mathbf{r}^{3}}
$$

The electric field excited by a permanent dipole $\boldsymbol{\mu}$ at the distance $\mathbf{r}$ is,

$$
\mathbf{E}_{\text {dipole }}(\mathbf{r})=-\nabla \phi_{\text {dipole }}(\mathbf{r})=\frac{1}{4 \pi \varepsilon_{0}}\left(\frac{3(\boldsymbol{\mu} \cdot \mathbf{r}) \mathbf{r}}{\mathbf{r}^{5}}-\frac{\boldsymbol{\mu}}{\mathbf{r}^{3}}\right)
$$

\section{Potential of quadrupole in static electric field}

The potential of a quadrupole in electric field $\mathbf{F}$ is the double point product of two tensors,

$$
\mathrm{U}=-\frac{1}{3} \boldsymbol{\Theta}: \mathbf{\nabla F}
$$

where $\boldsymbol{\Theta}$ is the quadrupole moment tensor and $\boldsymbol{\nabla F}$ is the EFG tensor.

\section{Electric field generated by a quadrupole}

The electric potential of elementary charge in the electric field excited by a quadrupole $\Theta$ at the distance $\mathbf{r}$ is,

$$
\phi_{\text {quadrupole }}(\mathbf{r})=\frac{1}{4 \pi \varepsilon_{0}} \frac{1}{2} \frac{\mathbf{r}^{\mathbf{T}} \cdot \boldsymbol{\Theta} \cdot \mathbf{r}}{\mathbf{r}^{5}}
$$

The electric field excited by a quadrupole $\boldsymbol{\Theta}$ at the distance $\mathbf{r}$ is,

$$
\mathbf{E}_{\text {quadrupole }}(\mathbf{r})=-\nabla \phi_{\text {quadrupole }}(\mathbf{r})=\frac{1}{4 \pi \varepsilon_{0}}\left(\frac{\left(5 \mathbf{r}^{\mathrm{T}} \cdot \boldsymbol{\Theta} \cdot \mathbf{r}\right) \mathbf{r}}{2 \mathbf{r}^{7}}-\frac{\boldsymbol{\Theta} \cdot \mathbf{r}}{\mathbf{r}^{5}}\right)
$$

The EFG tensor of quadrupole $\boldsymbol{\Theta}$ at the distance $\mathbf{r}$ is 


$$
\mathbf{E F G}(\mathbf{r})=\nabla \mathbf{E}_{\text {quadrupole }}(\mathbf{r})=\frac{1}{4 \pi \varepsilon_{0}}\left(\frac{\left(5 \mathbf{r}^{\mathbf{T}} \cdot \boldsymbol{\Theta} \cdot \mathbf{r}\right) \mathbf{I}}{2 \mathbf{r}^{7}}+\frac{\boldsymbol{\Theta}}{\mathbf{r}^{5}}\right)
$$

where $\mathbf{r}^{\mathbf{T}}$ is the transposed position vector, $\mathbf{I}$ is a $3 \times 3$ unit diagonal matrix.

\section{Computational strategy for electric field, interaction potential and isosteric heat}

The computational strategy for electric field generated by both molecules of this simulation is shown in Table 1S.

Table 1S. The computation of electric field with induction effect for a pair of molecules

Step 0: Initiate electric fields

$$
\begin{aligned}
& \mathbf{E}_{\mathbf{m}}(-\mathbf{r})=\mathbf{E}_{\mathbf{m}}^{0}(-\mathbf{r})=\frac{1}{4 \pi \varepsilon_{0}}\left(\frac{\boldsymbol{\mu}_{\mathbf{m}}}{\mathbf{r}^{3}}-\frac{3\left(\boldsymbol{\mu}_{\mathbf{m}} \cdot \mathbf{r}\right) \mathbf{r}}{\mathbf{r}^{5}}\right)+\frac{1}{4 \pi \varepsilon_{0}}\left(\frac{\left(5 \mathbf{r}^{\mathbf{T}} \cdot \boldsymbol{\Theta}_{\mathbf{m}} \cdot \mathbf{r}\right) \mathbf{r}}{2 \mathbf{r}^{7}}-\frac{\boldsymbol{\Theta}_{\mathbf{m}} \cdot \mathbf{r}}{\mathbf{r}^{5}}\right) \\
& \mathbf{E}_{\mathbf{M}}(\mathbf{r})=\mathbf{E}_{\mathbf{M}}^{0}(\mathbf{r})=\frac{1}{4 \pi \varepsilon_{0}}\left(\frac{\left(5 \mathbf{r}^{\mathbf{T}} \cdot \boldsymbol{\Theta}_{\mathbf{M}} \cdot \mathbf{r}\right) \mathbf{r}}{2 \mathbf{r}^{7}}-\frac{\boldsymbol{\Theta}_{\mathbf{M}} \cdot \mathbf{r}}{\mathbf{r}^{5}}\right) \\
& \mathbf{E}_{\mathbf{m}}^{\mathrm{I}}(-\mathbf{r})=\left[\mathbf{E}_{\mathbf{m}}^{\mathrm{I}}(-\mathbf{r})\right]^{0}=0 \\
& \mathbf{E}_{\mathbf{M}}^{\mathrm{I}}(\mathbf{r})=\left[\mathbf{E}_{\mathbf{M}}^{\mathrm{I}}(\mathbf{r})\right]^{0}=0 \\
& \mathrm{i}=1
\end{aligned}
$$

Step 1:Update electric fields

$$
\begin{aligned}
& \mathbf{E}_{\mathbf{m}}^{\mathrm{i}}(-\mathbf{r})=\mathbf{E}_{\mathbf{m}}^{0}(-\mathbf{r})+\mathbf{E}_{\mathbf{m}}^{\mathrm{I}}(-\mathbf{r}) \\
& \mathbf{E}_{\mathbf{M}}^{\mathrm{i}}(\mathbf{r})=\mathbf{E}_{\mathbf{M}}^{0}(\mathbf{r})+\mathbf{E}_{\mathbf{M}}^{\mathrm{I}}(\mathbf{r})
\end{aligned}
$$

Step2: Calculate induced dipoles

$$
\begin{aligned}
& \boldsymbol{\mu}_{\mathbf{m}}^{\mathrm{I}}=\boldsymbol{\alpha}_{\mathbf{m}} \cdot \mathbf{E}_{\mathbf{M}}^{\mathrm{i}}(\mathbf{r}) \\
& \boldsymbol{\mu}_{\mathbf{M}}^{\mathrm{I}}=\boldsymbol{\alpha}_{\mathbf{M}} \cdot \mathbf{E}_{\mathbf{m}}^{\mathrm{i}}(-\mathbf{r})
\end{aligned}
$$

Step3: Update electric fields of induced dipoles

$$
\begin{aligned}
& \mathbf{E}_{\mathbf{m}}^{\mathrm{I}}(-\mathbf{r})=\left[\mathbf{E}_{\mathbf{m}}^{\mathrm{I}}(-\mathbf{r})\right]^{\mathrm{i}}=\frac{1}{4 \pi \varepsilon_{0}}\left(\frac{\boldsymbol{\mu}_{\mathbf{m}}^{\mathrm{I}}}{\mathbf{r}^{3}}-\frac{3\left(\boldsymbol{\mu}_{\mathbf{m}}^{\mathrm{I}} \cdot \mathbf{r}\right) \mathbf{r}}{\mathbf{r}^{5}}\right) \\
& \mathbf{E}_{\mathbf{M}}^{\mathrm{I}}(\mathbf{r})=\left[\mathbf{E}_{\mathbf{M}}^{\mathrm{I}}(\mathbf{r})\right]^{\mathrm{i}}=\frac{1}{4 \pi \varepsilon_{0}}\left(\frac{3\left(\boldsymbol{\mu}_{\mathbf{M}}^{\mathrm{I}} \cdot \mathbf{r}\right) \mathbf{r}}{\mathbf{r}^{5}}-\frac{\boldsymbol{\mu}_{\mathbf{M}}^{\mathrm{I}}}{\mathbf{r}^{3}}\right)
\end{aligned}
$$


Step 4: Iteration*

$$
\text { if }\left(\left|\mathbf{E}_{\mathbf{m}}^{\mathrm{i}}(-\mathbf{r})-\mathbf{E}_{\mathbf{m}}^{\mathrm{i}-1}(-\mathbf{r})\right| /\left|\mathbf{E}_{\mathbf{m}}^{\mathrm{i}-1}(-\mathbf{r})\right|<\mathbf{e} \text { and }\left|\mathbf{E}_{\mathbf{M}}^{\mathrm{i}}(\mathbf{r})-\mathbf{E}_{\mathbf{M}}^{\mathrm{i}-1}(\mathbf{r})\right| /\left|\mathbf{E}_{\mathbf{M}}^{\mathrm{i}-1}(\mathbf{r})\right|<\mathbf{e}\right)
$$

Stop computation

else

$$
\mathrm{i}=\mathrm{i}+1 \text { and Go to Step } 1
$$

*The total number of iterations can be defined to stop the computation as long as the difference of electric field calculated in two consecutive iterations satisfies the convergence criterion $\boldsymbol{e}$.

The sum of electric fied of all pairs of the adsorbate and adsorbent molecules can be used to calculate the induction potential of the whole system.

The computational strategy for interaction potential of one adsorbate molecule and the multilayers of carbon atoms is shown in Table $2 \mathbf{S}$.

Table 2S. The computation of interaction potential of one adsorbate molecule and multilayer carbon atoms

Step 0: Initiate position and orientation of adsorbate molecule

$$
\begin{aligned}
& \mathbf{r}_{\mathbf{M}}=\mathbf{T}_{\boldsymbol{\alpha}, \boldsymbol{\beta}, \boldsymbol{\gamma}} \mathbf{r}_{\mathbf{m}}+\mathbf{r}_{\mathbf{m}, \mathbf{0}} * \\
& \mathrm{U}_{\mathrm{mM}}=\mathrm{U}_{\mathrm{LJ}}^{0}=\mathrm{U}_{\mathrm{E}}^{0}=\mathrm{U}_{\mathrm{I}}^{0}=0 \\
& \mathrm{i}=1
\end{aligned}
$$

Step 1:Update electric field, EFG and induced dipole moment Update $\mathbf{E}_{\mathbf{m}}^{\mathrm{i}}\left(-\mathbf{r}_{\mathbf{M}}\right), \mathbf{E}_{\mathbf{M}}^{\mathrm{i}}\left(\mathbf{r}_{\mathbf{M}}\right), \boldsymbol{\mu}_{\mathbf{m}}^{\mathrm{I}}$ and $\boldsymbol{\mu}_{\mathbf{M}}^{\mathrm{I}}$ according to Table 1

$$
\operatorname{EFG}_{\mathbf{M}}^{\mathrm{i}}\left(\mathbf{r}_{\mathbf{M}}\right)=\frac{1}{4 \pi \varepsilon_{0}}\left(\frac{\left(5 \mathbf{r}_{\mathbf{M}}{ }^{\mathbf{T}} \cdot \boldsymbol{\Theta} \cdot \mathbf{r}_{\mathbf{M}}\right) \mathbf{I}}{2 \mathbf{r}_{\mathbf{M}}{ }^{7}}+\frac{\boldsymbol{\Theta}}{\mathbf{r}_{\mathbf{M}}{ }^{5}}\right)
$$

Step2: Calculate interaction potential

$$
\begin{aligned}
& \mathrm{U}_{\mathrm{LJ}}^{\mathrm{i}}=\mathrm{U}_{\mathrm{LJ}}^{\mathrm{i}-1}+\sum \mathrm{U}_{\mathrm{LJ}}\left(\mathbf{r}_{\mathbf{M}}\right) * * \\
& \mathrm{U}_{\mathrm{E}}^{\mathrm{i}}=\mathrm{U}_{\mathrm{E}}^{\mathrm{i}-1}+\boldsymbol{\mu}_{\mathbf{m}} \mathbf{E}_{\mathbf{M}}^{\mathrm{i}}\left(\mathbf{r}_{\mathbf{M}}\right)+\frac{1}{3} \boldsymbol{\Theta}_{\mathbf{m}}: \mathbf{E F G}_{\mathbf{M}}^{\mathrm{i}}\left(\mathbf{r}_{\mathbf{M}}\right)
\end{aligned}
$$




$$
\mathrm{U}_{\mathrm{I}}^{\mathrm{i}}=\mathrm{U}_{\mathrm{I}}^{\mathrm{i}-1}-\frac{1}{2} \boldsymbol{\mu}_{\mathbf{m}}^{\mathrm{I}} \mathbf{E}_{\mathrm{M}}^{\mathrm{i}}\left(\mathbf{r}_{\mathbf{M}}\right)-\frac{1}{2} \boldsymbol{\mu}_{\mathrm{M}}^{\mathrm{I}} \mathbf{E}_{\mathbf{m}}^{\mathrm{i}}\left(-\mathbf{r}_{\mathbf{M}}\right)
$$

Step 3: Iteration util I reaches the total number of carbon atoms

$$
\begin{aligned}
& \text { if }(\mathbf{i}>\mathbf{N}) \\
& U_{m M}=U_{L J}^{N}+U_{E}^{N}+U_{I}^{N}
\end{aligned}
$$

Stop computation

else

$$
\mathrm{i}=\mathrm{i}+1 \text { and Go to Step } 1
$$

\section{* See 9. Coordinate transformation}

**eq (1) in main text is applied to each atom of adsorbate molecule with corresponding position vectors and the sum will be the LJ part of interaction potential between one adsorbate molecule and one carbon atom.

The computational strategy for isosteric heat of adsorption at zero surface coverage is shown in

\section{Table 3S.}

Table 3S. The computation of isosteric heat of adsorption at zero surface coverage

Step 0: Initialize constant and parameters

$\mathrm{k}_{B}, \mathrm{~T}$, integration method and precision, etc

Step 1: Initialize interaction potential function*

$$
\begin{aligned}
& \mathrm{U}_{\mathrm{mM}}(\mathrm{z})=\left.\mathrm{U}_{\mathrm{mM}}(\mathbf{r}(\mathrm{z}))\right|_{\mathrm{x}_{0}, \mathrm{y}_{0}}=\mathrm{U}_{\mathrm{mM}}\left(\mathrm{x}_{0}, \mathrm{y}_{0}, \mathrm{z}\right) \\
& \mathrm{U}_{\mathrm{mM}}(\mathrm{H}-\mathrm{z})=\left.\mathrm{U}_{\mathrm{mM}}(\mathrm{r}(\mathrm{H}-\mathrm{z}))\right|_{\mathrm{x}_{0}, \mathrm{y}_{0}}=\mathrm{U}_{\mathrm{mM}}\left(\mathrm{x}_{0}, \mathrm{y}_{0}, \mathrm{H}-\mathrm{z}\right)
\end{aligned}
$$

Step 2: Initialize external wall potential

$$
\mathrm{V}_{\mathrm{ext}}(\mathrm{z})=\mathrm{U}_{\mathrm{mM}}(\mathrm{z})+\mathrm{U}_{\mathrm{mM}}(\mathrm{H}-\mathrm{z})=\mathrm{U}_{\mathrm{mM}}\left(\mathrm{x}_{0}, \mathrm{y}_{0}, \mathrm{z}\right)+\mathrm{U}_{\mathrm{mM}}\left(\mathrm{x}_{0}, \mathrm{y}_{0}, \mathrm{H}-\mathrm{z}\right)
$$

Step 3: Integerate for numerator

$$
f_{\text {nume }}(\mathrm{H})=\int_{0}^{H} \mathrm{~V}_{\text {ext }}(\mathrm{z}) e^{-\frac{\mathrm{V}_{\text {ext }}(\mathrm{z})}{\mathrm{k}_{B} \mathrm{~T}}} d z
$$

Step 4: Integrate for denominator 


$$
f_{\text {denomi }}(\mathrm{H})=\int_{0}^{H} e^{-\frac{\mathrm{V}_{\text {ext }}(\mathrm{z})}{\mathrm{k}_{B} \mathrm{~T}}} d z
$$

Step 5: Calculate isosteric heat of adsorption

$$
q_{s t}^{o}(\mathrm{H})=\mathrm{k}_{B} \mathrm{~T}-\frac{\int_{0}^{H} \mathrm{~V}_{\text {ext }}(\mathrm{z}) e^{-\frac{\mathrm{V}_{\mathrm{ext}}(\mathrm{z})}{\mathrm{k}_{B} \mathrm{~T}}} d z}{\int_{0}^{H} e^{-\frac{\mathrm{V}_{\mathrm{ext}}(\mathrm{z})}{\mathrm{k}_{B} \mathrm{~T}}} d z}=\mathrm{k}_{B} \mathrm{~T}-\frac{f_{\text {nume }}(\mathrm{H})}{f_{\text {denomi }}(\mathrm{H})}
$$

Stop computation.

* The interation potential as a function of separation distance $\mathrm{z}$ is obtained by interpolation method from simulation data provided in Table 2S.

\section{Dipole-quadrupole interaction potential}

Non-zero dipole and quadrupole moment exists in the interaction pair of molecule (denoted by $\mathbf{m}$ and $\mathbf{M}$, respectively), the dipole-quadrupole interaction needs to be taken into consideration in the calculation of interaction potential. The interaction effect may decompose into three steps, i.e.,

firstly, the generation of electric field by the dipole and quadrupole,

secondly, the deriving of interaction potential of dipole and quadrupole in the generated static electric field (electrostatic potential),

thirdly, the deriving of interaction potential of induced dipole in the generated static electric field (induction potential).

Therefore, the dipole-quadrupole interaction consist of electrostatic potential $\mathrm{u}_{\mathrm{E}}$ and induction potential $\mathrm{u}_{\mathrm{I}}$. The electrostatic potential is,

$$
\begin{aligned}
\mathrm{U}_{\mathrm{E}} & =\mathrm{U}_{\text {dipole }, \mathrm{E}(\text { quarupole } \rightarrow \text { dipole })}+\mathrm{U}_{\text {quadrupole }, \mathrm{E}(\text { dipole } \rightarrow \text { quarupole })} \\
& =\boldsymbol{\mu}_{\mathbf{m}} \cdot \mathbf{E}_{\mathbf{M}}(\mathbf{r})+\frac{1}{3} \boldsymbol{\Theta}_{\mathbf{m}} \cdot \boldsymbol{\nabla} \mathbf{E}_{\mathbf{M}}(\mathbf{r})
\end{aligned}
$$


where $\mathbf{r}$ is the distance vector from quadrupole to dipole, $\mathbf{E}_{\mathbf{M}}(\mathbf{r})$ is the electric filed at the dipole generated by the quadrupole, $\nabla \mathbf{E}_{\mathbf{M}}(\mathbf{r})$ is the $\mathbf{E F G}$ of $\mathbf{E}_{\mathbf{M}}(\mathbf{r})$. Considering that the molecule $M$ with only non-zero quadrupole moment can still induce dipole in static electric field, both induction effects should be added to the total induction potential during deriving $\mathrm{U}_{\mathrm{I}}$. The induction potential is

$$
\begin{aligned}
\mathrm{U}_{\mathrm{I}} & =\mathrm{U}_{\text {dipole, } \mathrm{E}(\text { quarupole } \rightarrow \text { dipole })}^{\mathrm{I}}+\mathrm{U}_{\text {quadrupole }, \mathrm{E}(\text { dipole } \rightarrow \text { quarupole })}^{\mathrm{I}} \\
& =-\frac{1}{2} \boldsymbol{\alpha}_{\mathrm{m}} \cdot \mathbf{E}_{\mathrm{M}}^{2}(\mathbf{r})-\frac{1}{2} \boldsymbol{\alpha}_{\mathrm{M}} \cdot \mathbf{E}_{\mathbf{m}}^{2}(-\mathbf{r})
\end{aligned}
$$

where $\boldsymbol{\alpha}_{\mathrm{m}}$ is the dipole polarizability of the molecule with non-zero dipole moment $\boldsymbol{\mu}, \boldsymbol{\alpha}_{\mathrm{M}}$ is the dipole polarizability of the molecules with non-zero quadrupole moment $\boldsymbol{\Theta}$. In consideration of the contribution of electric field generated by the induced dipole on molecules, the electric field at $\mathbf{r}$ and $-\mathbf{r}$ should be calculated iteratively.

\section{Interaction Potential of Water on Graphite}

With fixed $\mathrm{z}$, the interaction potential can vary with $\mathrm{x}$ and $\mathrm{y}$ because of the change of relative orientation of adsorbate molecule to the center of hexagonal unit cell of graphite lattice. The distribution of interaction potential of water on graphite at orientation 1 to 5 in the simulated space is plotted as Figures S1(a) to S1(e). Six blue points represent the position of carbon atoms of hexagonal unit cell on XY plane. 


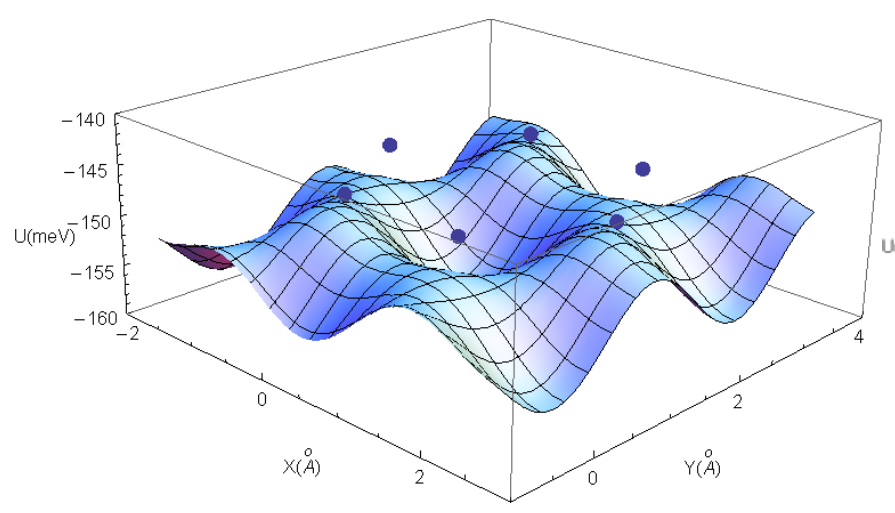

Figure S1(a): Interaction potential of water on graphite at orientation 1 and $Z=3 \AA$.

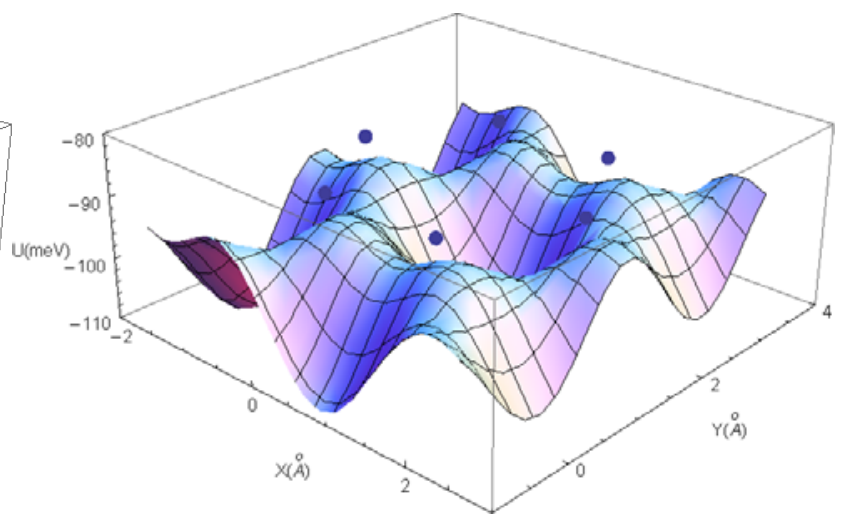

Figure S1(b): Interaction potential of water on graphite at orientation 2 and $\mathrm{Z}=3.5 \AA$.

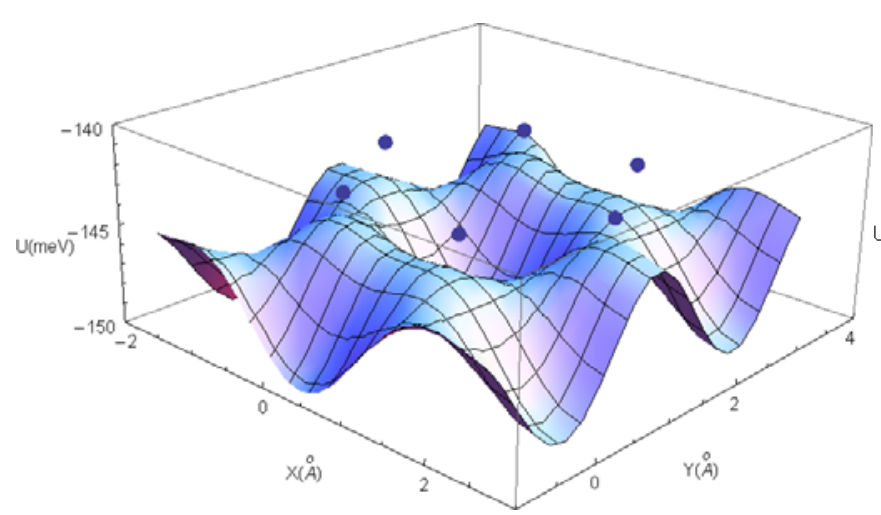

Figure $S 1(c)$ : Interaction potential of water on graphite at orientation 3 and $Z=3 \AA$

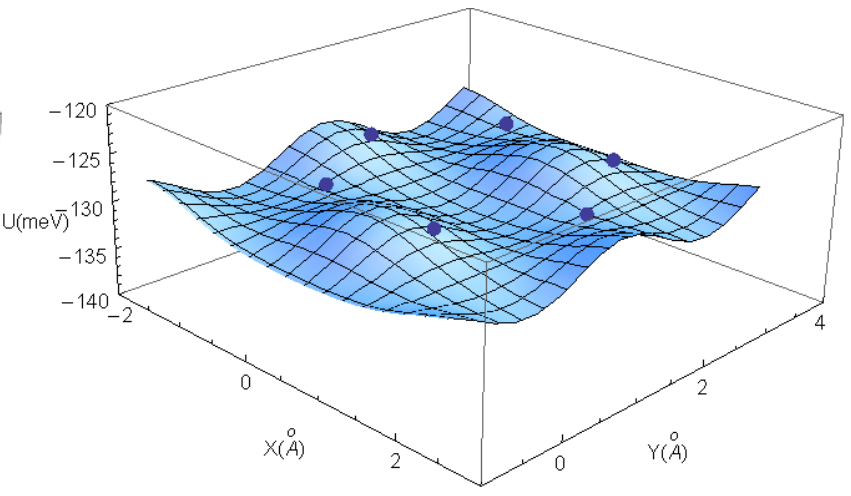

Figure S1(d): Interaction potential of water on graphite at orientation 4 and $Z=3.5 \AA$.

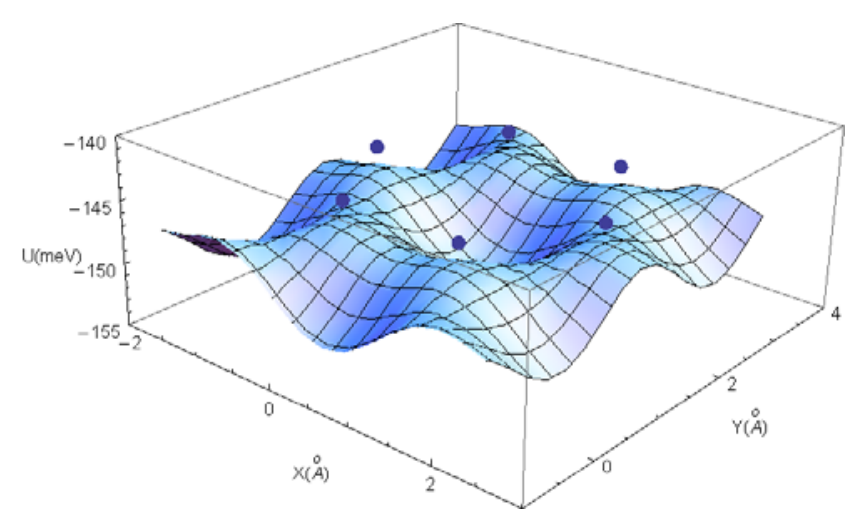

Figure S1(e): Interaction potential of water on graphite at orientation 5 and $Z=3 \AA$. 
For understanding the LJ, induction and electrostatic potentials, plots of interaction energy as a function of $\mathrm{C} \cdots \mathrm{O}$ separation distance for orientations 4 and 5 of a water molecule are shown in Figures S2(a) and (b).
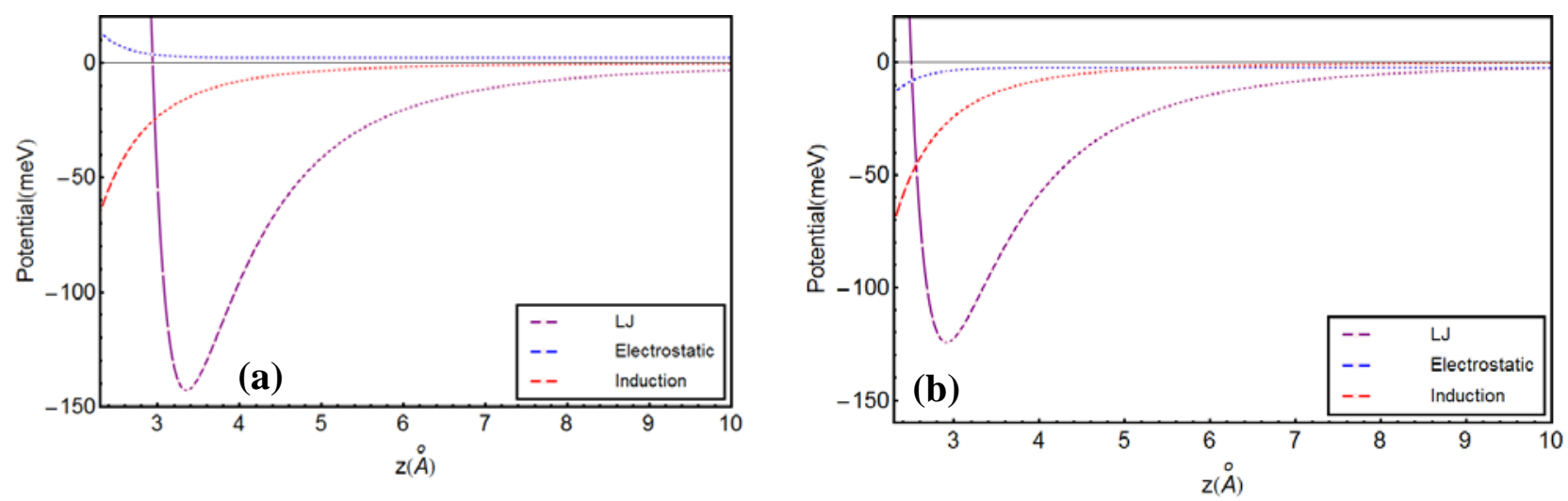

Figure S2: Water potential plots for (a) orientation 4 (b) orientation $5\left(\mathrm{U}_{\mathrm{E}}>0\right.$ for orientation 4 and $\mathrm{U}_{\mathrm{E}}<0$ for orientation 5)

\section{Interaction Potential of Ammonia on Graphite}

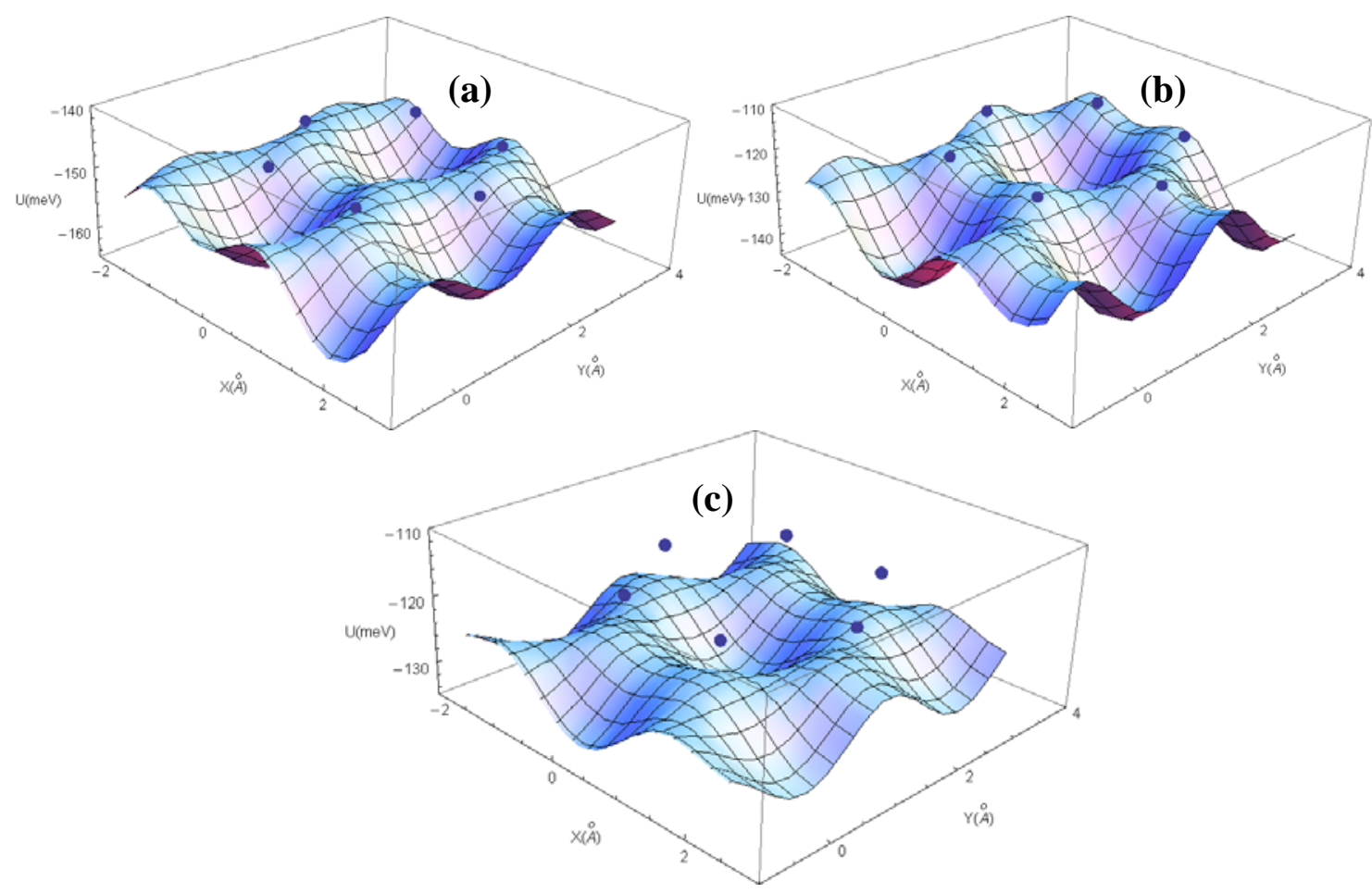

Figure S3: Interaction potentials of ammonia on graphite at (a) orientation 1 for $\mathrm{Z}=3.5 \AA$, (b) orientation 2 and $\mathrm{Z}=3.5 \AA$, and (c) orientation 3 and $\mathrm{Z}=3.5 \AA$. 
The distribution of interaction potential of ammonia on graphite at orientations 1 to 3 in the simulated space is plotted in Figures S3(a) to S3(c). Six blue points represent the orientation of carbon atoms of hexagonal unit cell on XY plane. Similarly, for understanding the LJ, induction and electrostatic potentials separately, plots of interaction energy as a function of $\mathrm{C}^{\cdots} \mathrm{NH}_{3}$ separation distance for orientations 2 and 3 of an ammonia molecule are shown in Figures S4(a) and (b).
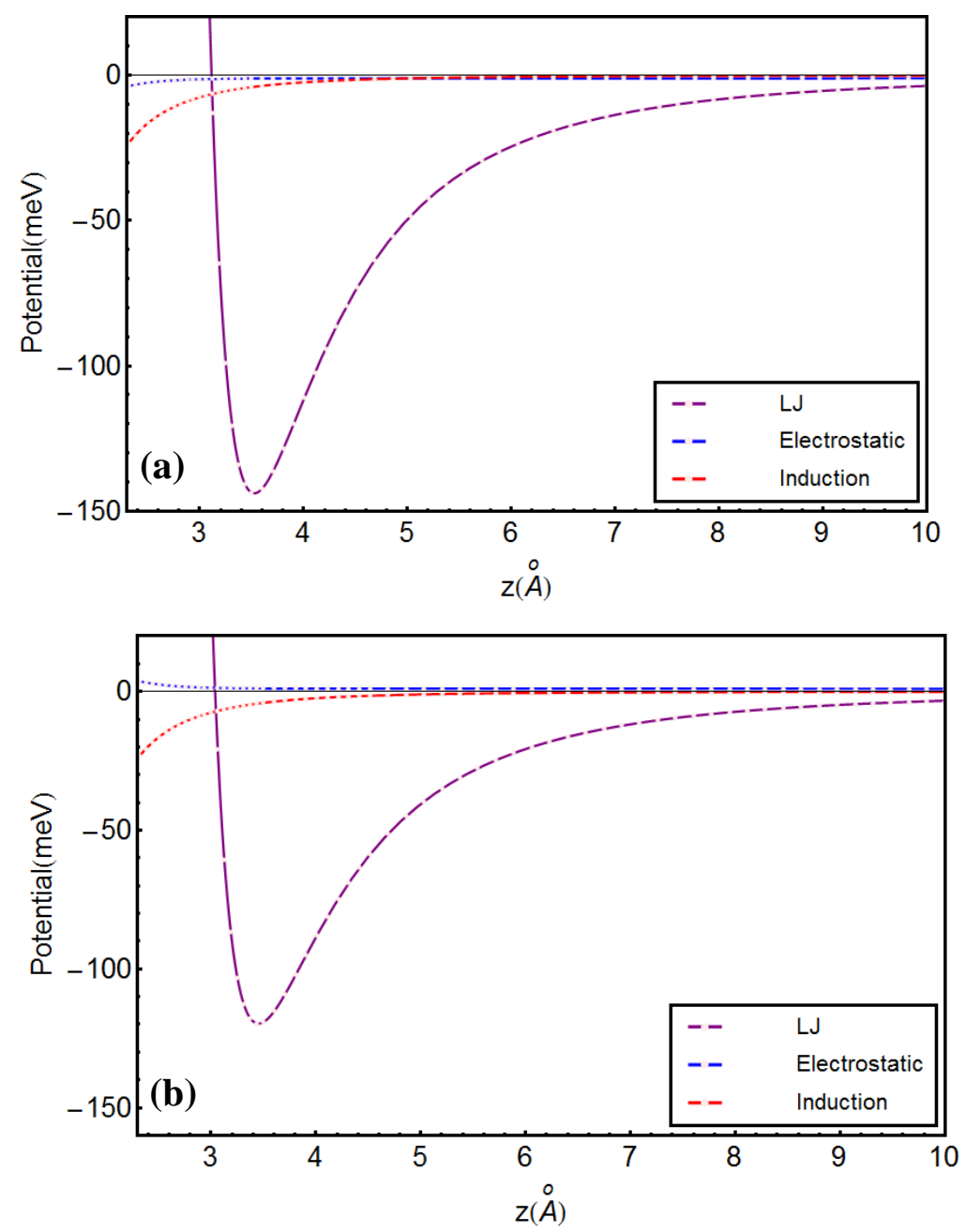

Figure S4: Ammonia interaction potentials on graphite for (a) orientation 2 and (b) orientation $3\left(\mathrm{U}_{\mathrm{E}}>0\right.$ for orientation 3 and $\mathrm{U}_{\mathrm{E}}<0$ for orientation 2) 


\section{Interaction Potential of Methanol on Graphite}

The distribution of interaction potential of methanol on graphite at orientations 1, 2 and 3 in the simulated space are plotted in Figures S5(a), S5(b) and S5(c). Six blue points represent the orientation of carbon atoms of hexagonal unit cell on XY plane.

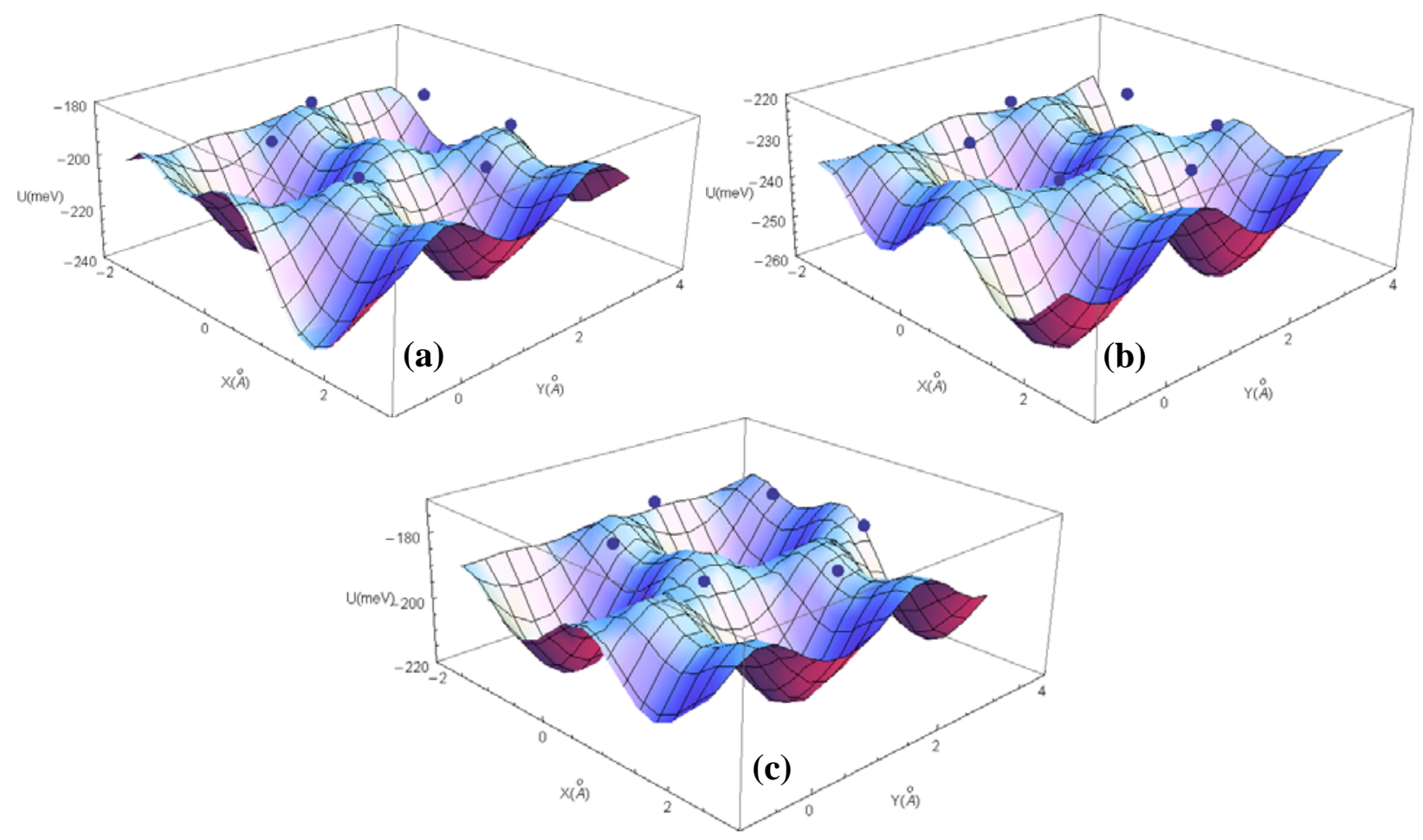

Figure S5: Interaction potential of methanol molecules on graphite for orientations 1,2 and 3 at $3.5 \AA$.

For better understanding the LJ, induction and electrostatic potentials separately, a plot of interaction energy as a function of $\mathrm{C} \cdots \mathrm{CH}_{3}-\mathrm{OH}$ separation distance for orientation 3 of ammonia molecule is shown in Figure S6. 


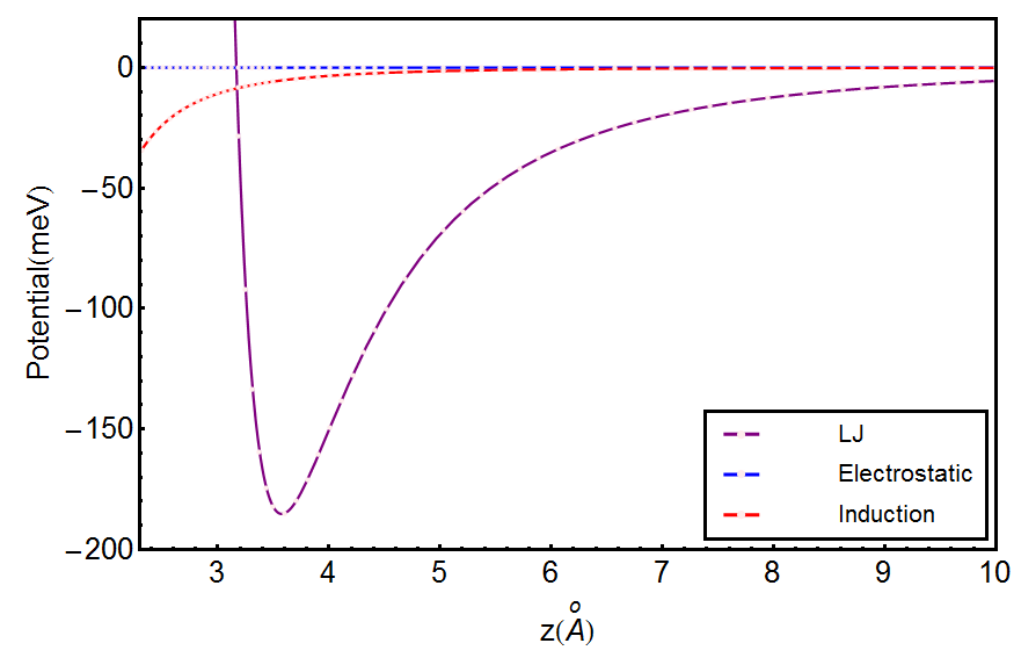

Figure S6: Various interaction energy for methanol and graphite system at orientation 3

( $\mathrm{U}_{\mathrm{E}}<0$ for all of the 3 orientations)

\section{Interaction Potential of Ethanol on Graphite}

The distribution of interaction potential of ethanol on graphite ranging from orientations 1 to 3 in the simulated space is plotted in Figures S7(a), S7(b) and S7(c). Six blue points represent the position of carbon atoms of hexagonal unit cell on XY plane.

The $\mathrm{LJ}$, induction and electrostatic potentials are plotted as a function of $\mathrm{C} \cdots \mathrm{CH}_{3}-\mathrm{CH}_{2}-\mathrm{OH}$ separation distance for the orientation 2 of ammonia molecule is shown in Figure S8. It is observed that the electrostatic potential is very closed to zero and provides attractive force. 


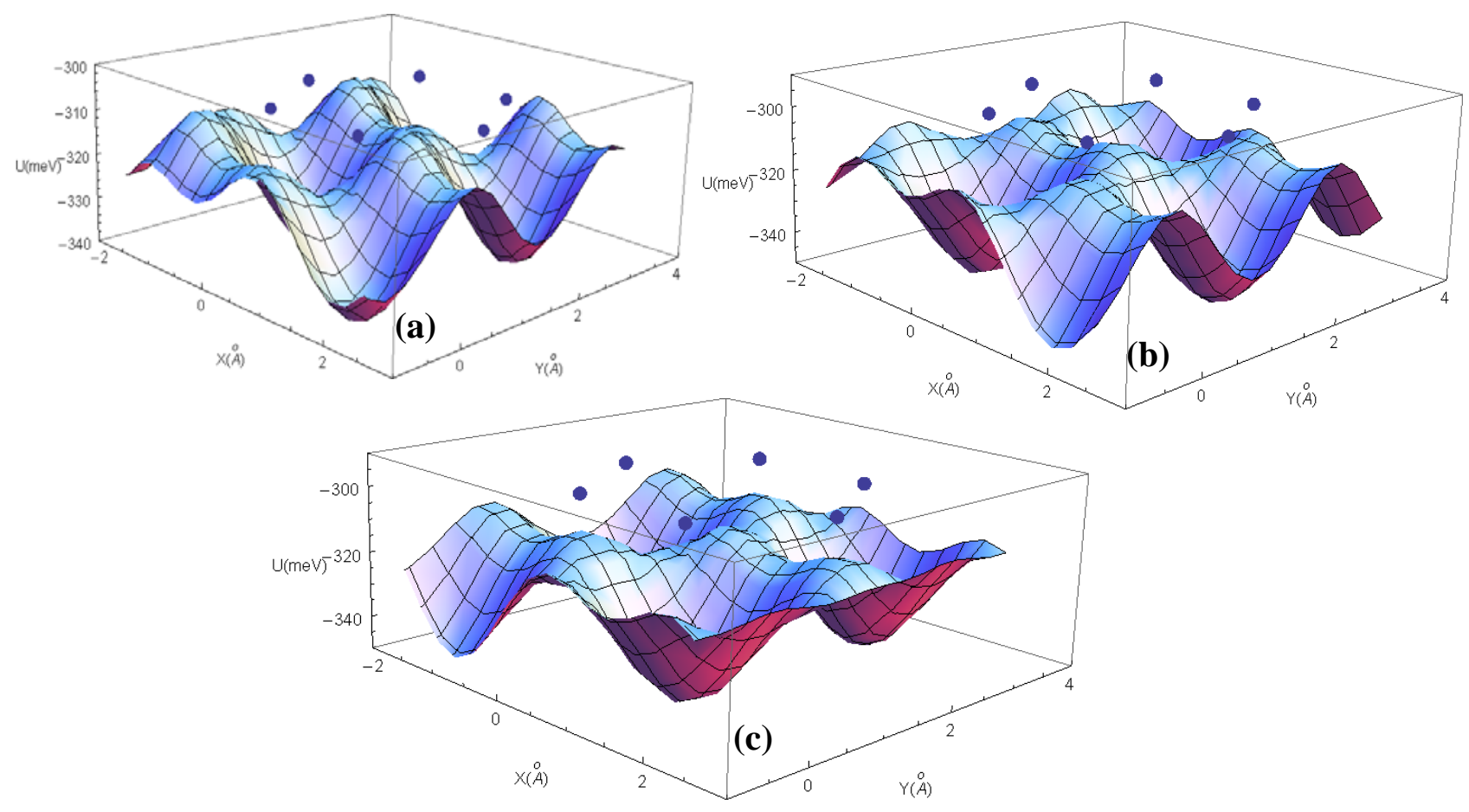

Figure S7: Interaction potential surface of ethanol on graphite for (a) orientation 1 (b) orientation 2 and (c) orientation 3 at $Z=3.5 \AA$.

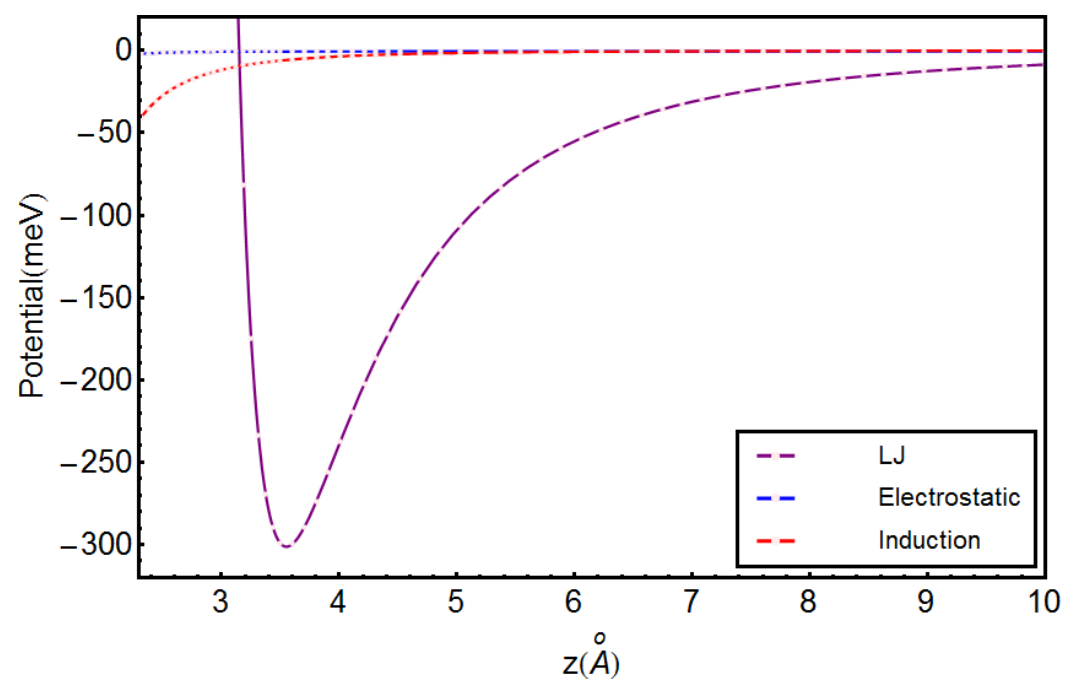

Figure S8: Ethanol potential for orientation 2 ( $\mathrm{U}_{\mathrm{E}}<0$ for all of the 3 orientations) 


\section{Coordinate Transformation}

For coordinate of atoms of adsorbate molecule, the coordinate transformation from the adsorbate coordinate system $\left(\mathrm{X}^{\prime} \mathrm{Y}^{\prime} \mathrm{Z}^{\prime}\right)$ to the adsorbent (graphite) coordinate system (XYZ) can be accomplished by rotation and translation.

The column vector $\left(x^{\prime}, y^{\prime}, z^{\prime}\right)$ in $\mathrm{X}^{\prime} \mathrm{Y}^{\prime} \mathrm{Z}^{\prime}$ system is rotated in the order of around $\mathrm{X}^{\prime}, \mathrm{Y}^{\prime}$ and $\mathrm{Z}^{\prime}$ axis by $\alpha, \beta$ and $\gamma$ degree correspondingly. This temporary vector is then translated by a given translational vector $\left(x_{0}, y_{0}, z_{0}\right)$ and the final column vector $(x, y, z)$ is the transformation result from X'Y'Z' system to XYZ system.

$\left[\begin{array}{l}x \\ y \\ z\end{array}\right]=\left[\begin{array}{ccc}\cos \beta \cos \gamma & \cos \gamma \sin \alpha \sin \beta-\cos \alpha \sin \gamma & \cos \alpha \cos \gamma \sin \beta+\sin \alpha \sin \gamma \\ \cos \beta \sin \gamma & \sin \alpha \sin \beta \sin \gamma+\cos \alpha \cos \gamma & \cos \alpha \sin \beta \sin \gamma-\cos \gamma \sin \alpha \\ -\sin \beta & \cos \beta \sin \alpha & \cos \alpha \cos \beta\end{array}\right]\left[\begin{array}{l}x^{\prime} \\ y^{\prime} \\ z^{\prime}\end{array}\right]+\left[\begin{array}{l}x_{0} \\ y_{0} \\ z_{0}\end{array}\right]$

By combineing various rotational angles and translational vectors, the coordinate of atoms of adsorbate molecules in any angle and distance to adsorbent molecules can be obtained.

\section{Molecule Coordinate}

\section{Water}

3

$\mathrm{H}_{2} \mathrm{O}$

O $\quad 0.008165 \quad-0.658276 \quad 0.215737$

H $\quad 1.508729 \quad 0.441434 \quad 0.259102$

$\begin{array}{llll}\mathrm{H} & -1.419465 & 0.543189 & 0.243811\end{array}$

\section{Ammonia}

4

$\mathrm{NH}_{3}$

$\begin{array}{llll}\mathrm{N} & 0.257 & -0.363 & 0.000\end{array}$ 

$\begin{array}{llll}\mathrm{H} & 0.257 & 0.727 & 0.000\end{array}$
$\begin{array}{llll}\mathrm{H} & 0.771 & -0.727 & 0.890\end{array}$
H $\quad 0.771 \quad-0.727 \quad-0.890$

\begin{abstract}
Methanol
6

$\mathrm{CH}_{4} \mathrm{O}$

$\begin{array}{llll}\mathrm{O} & 0.7079 & 0.0000 & 0.0000\end{array}$

$\begin{array}{llll}\text { C } & -0.7079 & 0.0000 & 0.0000\end{array}$

H $\quad-1.0732 \quad-0.7690 \quad 0.6852$

$\mathrm{H} \quad-1.0731 \quad-0.1947 \quad-1.0113$

$\begin{array}{llll}\mathrm{H} & -1.0632 & 0.9786 & 0.3312\end{array}$

H $\quad 0.9936 \quad-0.8804 \quad-0.2980$
\end{abstract}

\title{
Ethanol
}

9

$\mathrm{C}_{2} \mathrm{H}_{6} \mathrm{O}$

$\begin{array}{llll}\text { H } & -2.0801425360 & 0.4329727646 & 0.0722817289\end{array}$

C $\quad-1.2129704155 \quad-0.2295285634 \quad-0.0097156258$

$\mathrm{H} \quad-1.2655910941 \quad-0.9539857247 \quad 0.8097953440$

$\begin{array}{llll}\text { C } & 0.0849758188 & 0.5590385475 & 0.0510545434\end{array}$

$\begin{array}{llll}\text { O } & 1.2322305822 & -0.2731895077 & -0.1276123902\end{array}$

$\begin{array}{llll}\mathrm{H} & 0.1506137362 & 1.1200249874 & 0.9943015309\end{array}$

H $\quad 1.2473876659 \quad-0.8998737590 \quad 0.6150681570$

$\mathrm{H} \quad 0.1316093068 \quad 1.2841805400 \quad-0.7645223601$

$\mathrm{H} \quad-1.2737541560 \quad-0.7748626513 \quad-0.9540587845$ 


\section{Nomenclatures}
Abbreviations
$\mathrm{q}_{\mathrm{st}}$
adsorption isosteric heat
EFG
electric field gradient
LJ
Lenard-Jones

\section{Symbols}

E

$\mathbf{E}_{\text {dipole }}$

$\mathbf{E}_{\text {quadrupole }}$

F

I

$\mathrm{m}$

M

$\mathbf{r}$

$\mathbf{r}^{\mathbf{T}}$

$\mathbf{r}_{\mathbf{i}}$

$\mathbf{r}_{\mathbf{j}}$

$\mathbf{r}_{\mathrm{ij}}$

U

$\mathrm{U}_{\mathrm{LJ}}$

$\mathrm{U}_{\mathrm{E}}$ electric field vector

electric field vector of a dipole

electric field vector of a quadrupole

electric field vector

$3 \times 3$ unit diagonal matrix

adsorbate (water, ammonia, etc.)

adsorbent (graphite)

position vector between two multipole

transposed position vector

position vector of the $i$ th atom of the adsorbate molecule

position vector of the $j$ th carbon atom

position vector from the $i$ th carbon to the $j$ th atom of the

adsorbate molecule

potential

Lenard-Jones potential

electrostatic potential 


\begin{tabular}{ll}
$\mathrm{U}_{\mathrm{I}}$ & induction potential \\
$\boldsymbol{\alpha}$ & polarizability matrix \\
$\varepsilon_{0}$ & vacuum permittivity \\
$\varepsilon$ & potential \\
$\varepsilon_{s S}$ & adsorbent pair well depth potential \\
$\varepsilon_{f f}$ & adsorbate pair well depth potential \\
$\varepsilon_{s f}$ & pair well depth potential \\
$\boldsymbol{\Theta}$ & quadrupole moment matrix \\
$\boldsymbol{\mu}$ & dipole moment vector \\
$\boldsymbol{\mu}^{\mathrm{I}}$ & induced dipole moment vector \\
$\boldsymbol{\mu}_{\mathrm{H}_{2} \mathrm{O}}^{\mathrm{I}}$ & induced dipole moment vector of water molecule \\
$\boldsymbol{\mu}_{\mathrm{C}}^{\mathrm{I}}$ & induced dipole moment vector of carbon atom \\
$\sigma$ & collision diameter \\
$\sigma_{s s}$ & adsorbent collision diameter \\
$\sigma_{f f}$ & adsorbate collision diameter \\
$\sigma_{s f}$ & pair collision diameter \\
$\phi_{\mathrm{dipole}}$ & electrical potential of a dipole \\
$\phi_{\text {quadrupole }}$ & electrical potential of a quadrupole \\
\hline & \\
\hline &
\end{tabular}




\section{References}

(1) Buckingham, A. D. Permanent and Induced Molecular Moments and Long-Range Intermolecular Forces. Adv. Chem. Phys. 1967, 12, 107-142.

(2) McLean, A. D.; Yoshimine, M. Higher Polarizabilities of Linear Molecules. J. Chem. Phys. 1967, 46, 3682-3683.

(3) Maroulis, G.; Makris, C.; Xenides, D.; Karamanis, P. Electric dipole and quadrupole moment and dipole polarizability of CS, SiO and SiS. Mol. Phys. 2000, 98, 481-491.

(4) Pople, J. A. The Statistical Mechanics of Assemblies of Axially Symmetric Molecules. II. Second Virial Coefficients. Proc. R. Soc. London. Ser. A. 1954, 221, 508-516.

(5) Buckingham, A. D. Molecular quadrupole moments. Q. Rev. Chem. Soc. 1959, 13, 183-214.

(6) Griffiths, D. J. Introduction to Electodynamics; Prentice Hall: New Jersey, 1999. 